\title{
Expected Value and Chance Constrained Stochastic Unit Commitment Ensuring Wind Power Utilization
}

\author{
Chaoyue Zhao, Student Member, IEEE, Qianfan Wang, Student Member, IEEE, Jianhui Wang, Senior Member, IEEE, \\ and Yongpei Guan, Senior Member, IEEE
}

\begin{abstract}
This paper proposes an expected value and chance constrained stochastic optimization approach for the unit commitment problem with uncertain wind power output. In the model, the utilization of wind power can be adjusted by changing the utilization rate in the proposed expected value constraint. Meanwhile, the chance constraint is used to restrict the probability of load imbalance. Then a Sample Average Approximation (SAA) method is used to transform the objective function, the expected value constraint, and the chance constraint into sample average reformulations. Furthermore, a combined SAA framework that considers both the expected value and the chance constraints is proposed to construct statistical upper and lower bounds for the optimization problem. Finally, the performance of the proposed algorithm with different utilization rates and different risk levels is tested for a six-bus system. A revised IEEE 118-bus system is also studied to show the scalability of the proposed model and algorithm.
\end{abstract}

Index Terms - Chance constraint, expected value constraint, sample average approximation, stochastic optimization, unit commitment, wind power.

\section{NOMENCLATURE}

\section{A. Indices and Parameters}

$B \quad$ Index set of all buses.

BG Set of buses with thermal generation units.

BW Set of buses with wind farms.

$C_{i j} \quad$ Transmission capacity for the transmission line linking bus $i$ and bus $j$.

$D_{b t}(\xi) \quad$ Random parameter indicating the uncertain load at bus $b$ in time $t$ corresponding to scenario $\xi$.

$\mathrm{DR}_{i}^{b} \quad$ Ramp-down rate limit for generator $i$ at bus $b$.

Manuscript received May 31, 2013; revised October 06, 2013 and January 11, 2014; accepted March 08, 2014. Date of publication May 06, 2014; date of current version October 16, 2014. This work was supported in part by University of Chicago Argonne, LLC, Operator of Argonne National Laboratory ("Argonne"). Argonne, a U.S. Department of Energy Office of Science laboratory, is operated under Contract No. DE-AC02-06CH11357. Paper no. TPWRS00686-2013.

C. Zhao is with the Department of Industrial Engineering and Management, Oklahoma State University, Stillwater, OK 74074 USA (e-mail: cherryzhao09@gmail.com).

Q. Wang is with Alstom Grid, Redmond, WA 98052 USA (e-mail qfwang@ufl.edu).

J. Wang is with the Decision and Information Science Division, Argonne National Laboratory, Lemont, IL 60439 USA (e-mail: jianhui.wang@anl.gov).

Y. Guan is with the Department of Industrial and Systems Engineering, University of Florida, Gainesville, FL 32611 USA (e-mail: guan@ise.ufl.edu).

Color versions of one or more of the figures in this paper are available online at http://ieeexplore.ieee.org.

Digital Object Identifier 10.1109/TPWRS.2014.2319260
$F_{c}\left(q_{i t}^{b}(\xi)\right)$ Fuel cost of thermal generator $i$ at bus $b$ in time $t$ when its generation amount is $q_{i t}^{b}(\xi)$.

$K_{i j}^{b} \quad$ Line flow distribution factor for the transmission line linking bus $i$ and bus $j$, due to the net injection at bus $b$.

$L_{i}^{b} \quad$ Lower bound of electricity generated by thermal generator $i$ at bus $b$.

$M \quad$ Very big number.

$\mathrm{MD}_{i}^{b} \quad$ Minimum down-time for generator $i$ at bus $b$.

$\mathrm{MU}_{i}^{b} \quad$ Minimum up-time for generator $i$ at bus $b$.

$\mathrm{SD}_{i}^{b} \quad$ Shut-down cost of thermal generator $i$ at bus $b$.

$\mathrm{SU}_{i}^{b} \quad$ Start-up cost of thermal generator $i$ at bus $b$.

$T \quad$ Time horizon (e.g., 24 hours).

$U_{i}^{b} \quad$ Upper bound of electricity generated by thermal generator $i$ at bus $b$.

$\mathrm{UR}_{i}^{b} \quad$ Ramp-up rate limit for generator $i$ at bus $b$.

$W_{b t}(\xi) \quad$ A random parameter indicating the available wind power, or turbine capacity (decided by the weather) at bus $b$ in time $t$ corresponding to scenario $\xi$.

$\beta \quad$ Utilization rate of the wind power output.

$\Delta \quad$ Tolerance of load imbalance in the chance constraint.

$\epsilon \quad$ Risk level associated with the chance constraint on load imbalance.

$\gamma_{t}^{+} \quad$ Penalty cost per MWh for load curtailment in time $t$.

$\gamma_{t}^{-} \quad$ Penalty cost per MWh for over-generated power due to wind power and its uncertainty in time $t$.

$\Lambda_{b} \quad$ Set of thermal generators at bus $b$.

\section{B. First-Stage Decision Variables}

$o_{i t}^{b} \quad$ Binary decision variable: " 1 " if thermal generator $i$ at bus $b$ is on in time $t$; " 0 " otherwise.

$u_{i t}^{b} \quad$ Binary decision variable: " 1 " if thermal generator $i$ at bus $b$ is started up in time $t$; " 0 " otherwise.

$v_{i t}^{b} \quad$ Binary decision variable: " 1 " if thermal generator $i$ at bus $b$ is shut down in time $t$; " 0 " otherwise. 


\section{Second-Stage Decision Variables}

$q_{i t}^{b}(\xi) \quad$ Electricity generation amount by thermal generator $i$ at bus $b$ in time $t$ corresponding to scenario $\xi$.

$\hat{q}_{t}^{b}(\xi) \quad$ Amount of wind power utilized (delivered) at bus $b$ in time $t$ corresponding to scenario $\xi$.

$Q_{t}^{G}(\xi) \quad$ Total generation amount by thermal units in time $t$ corresponding to scenario $\xi$.

$Q_{t}^{I+}(\xi) \quad$ Total load curtailment in time $t$ corresponding to scenario $\xi$.

$Q_{t}^{I-}(\xi) \quad$ Total over-generated power in time $t$ corresponding to scenario $\xi$.

$Q_{t}^{W}(\xi) \quad$ Total wind power committed to be utilized (delivered) in time $t$ corresponding to scenario $\xi$.

\section{INTRODUCTION}

W ITH the explosive growth of available wind power, power system operation paradigms have faced profound challenges [1]. On one hand, government policies encourage the large scale penetration of wind power, which leads to positive impacts on greenhouse gas (GHG) reduction, water conservation, and energy security [2]. On the other hand, the forecast error of wind power introduces considerable uncertainties into the system operator's decision-making process. In particular, unit commitment (UC) and economic dispatch (ED) of conventional units are of great concern. These two well-known conventional power engineering practices have to accommodate wind power uncertainty to enable the high utilization of wind power.

To deal with the wind power output uncertainty in power system operations, two common methods have been studied extensively, deterministic reserve margins and stochastic programming.

The first method is to perform an accurate forecast and use the forecasted wind power output in UC and ED scheduling. The unexpected fluctuation of the real-time wind power output is compensated by the upward and downward reserves. The most important issue for this technique is to determine the optimal amount of reserves from both reliable and economic perspectives. Traditionally, deterministic criteria [3] were applied to decide the reserve level. Recently, probabilistic analysis [4] and stochastic optimization [5] have also been proposed to estimate the reserve requirement, in which the intermittent nature of wind power output is taken into account.

The second method is the stochastic optimization approach, for which researchers study wind power integration through solving different stochastic unit commitment formulations (see, e.g., [6]-[9] and [10], among others). Different approaches have been explored to solve corresponding formulations. For instance, in [11], an efficient Benders' decomposition approach is proposed to solve the security-constrained unit commitment (SCUC) problem involving the uncertainty and intermittency of wind power. In [12], both load and wind power uncertainties are considered, and an adaptive particle swarm optimization algorithm is applied to solve the stochastic unit commitment problem. In [13], a computational framework incorporating the Weather Research and Forecast (WRF) model is presented. In
[14], the interval optimization approach and the scenario-based stochastic programming method are compared to solve the stochastic SCUC with uncertain wind power output.

To maintain system reliability, wind power curtailment occurs frequently, resulting low utilization of wind power. Wind power curtailment can occur due to different reasons including transmission or distribution congestion, global oversupply, and operational issues. A 2010 report by the National Renewable Energy Laboratory (NREL) highlights the severity of wind power curtailment. For example, in Texas, the average annual wind power curtailment is as high as $16 \%$ of the total wind generation in 2009 [15]. The high wind power curtailment has big impacts on the economics of the wind power plant and will discourage the wind power investment to increase the usage of renewable energy. Therefore, the system operators are encouraged to utilize the wind power to its largest possible extent. To ensure high wind power utilization, the chance constrained stochastic optimization approach can be applied [16]. The chance constraints can be applied to improve the wind power penetration, with an explicit constraint on the probability of wind power utilization (see, e.g., [17], [18], and [19]). Note here that the chance constrained approach is also related to the risk-constrained stochastic optimization approach studied recently (see, e.g., [20], [21], and [22]), to assess and control the risk associated with the uncertainty. For instance, in [22], using the similar concept, the risk-aversion constraints are used to control generation company's financial risk under the consideration of uncertain wind power and electricity prices. This approach is also related to the robust optimization approach (see, e.g., [23]-[25], and [26]), which can be considered as a conservative approximation of the chance constrained approach by defining the uncertainty set such that the random variable locates in the uncertainty set with a certain probability.

Although high wind power utilization can be enforced in most scenarios through the chance-constrained stochastic optimization approach, the overall expected wind power utilization can be low. For instance, consider a case for which there is only one wind farm in the power system. The wind power output $W(\xi)$ follows a Bernoulli distribution with two possible outcomes: 1) $W(\xi)=W\left(\xi_{1}\right)=10 \mathrm{MW}$ with $90 \%$ of chance and 2) $W(\xi)=W\left(\xi_{2}\right)=100 \mathrm{MW}$ with $10 \%$ of chance. If there is only a chance constraint, e.g., $\operatorname{Pr}\left\{Q^{W}(\xi) \geq 80 \% W(\xi)\right\} \geq$ $90 \%$, to meet the constraint, the wind power can be utilized as $Q^{W}\left(\xi_{1}\right)=8 \mathrm{MW}$ and $Q^{W}\left(\xi_{2}\right)=0 \mathrm{MW}$. However, under this case, the total expected utilization of wind power is $8.90 \% /(10$. $90 \%+100 \cdot 10 \%)=37.8 \%$. As it can be seen, the chance constraint ignores the low-probability but high wind output scenario of $W\left(\xi_{2}\right)$, which may lead to insufficient wind power utilization. This low utilization of wind power can be avoided if an expected value constraint such as $E\left[Q^{W}(\xi)\right] \geq 80 \% E[W(\xi)]$ is added. Therefore, the chance constrained approach can ensure high wind power utilization for most outcomes, and the expected value constraint guarantees an overall high expected wind power utilization.

In this paper, both chance and expected value constraints are studied, in which the chance constraint enforces a low probability of load imbalance, and the expected value constraint ensures the overall expected amount of wind power usage. A related concept of the expected value constraint is the conditional value-at-risk (CVaR) decision criterion (see, e.g., [27], [28], and 
[29]), which is, as described in [30], a special case of the expected value constrained problem. Therefore, the method developed in this paper can also be applied to solve CVaR constrained stochastic unit commitment problems.

In this paper, a novel combined Sampling Average Approximation (SAA) method is developed to solve the problem. The main contributions of this paper are summarized as follows:

1) A novel stochastic unit commitment formulation is studied in which the expected value constraint is introduced to ensure wind power utilization.

2) A new combined SAA method, including theoretical convergence results and solution validation procedure, is developed, which can solve the stochastic unit commitment problems with both the chance and the expected value constraints.

The rest of this paper is organized as follows: Section II discusses the mathematical formulation and describes the expected value and chance constraints; Section III presents the solution methodology and describes the combined SAA algorithm; Section IV reports the computational experiments for a six-bus system and two revised 118-bus systems respectively; finally, Section V concludes the research.

\section{MATHEMATICAL Formulation}

In this section, a two-stage stochastic unit commitment formulation considering both the expected value and chance constraints is developed. The formulation captures both wind power output and load uncertainties. In the formulation, the first stage is to determine the day-ahead unit commitment decisions that include turn-on/turn-off decisions of thermal power generators. The second stage contains the decisions on the real-time dispatch of thermal units and the actual amount of wind power usage, depending on the random variable $\xi$. The penalty cost is introduced in the second stage for the load imbalance. In addition, there's no unit outage considered in this study. The detailed formulation is described as follows:

$$
\begin{aligned}
& \min \sum_{t=1}^{T} \sum_{b \in B} \sum_{i \in \Lambda_{b}}\left(\mathrm{SU}_{i}^{b} u_{i t}^{b}+\mathrm{SD}_{i}^{b} v_{i t}^{b}\right)+E[\mathcal{Q}(o, u, v, \xi)] \\
& \text { s.t. } \\
& -o_{i(t-1)}^{b}+o_{i t}^{b}-o_{i k}^{b} \leq 0 \\
& \forall k: 1 \leq k-(t-1) \leq \mathrm{MU}_{i}^{b}, \forall i \in \Lambda_{b}, \forall b \in B, \forall t \\
& o_{i(t-1)}^{b}-o_{i t}^{b}+o_{i k}^{b} \leq 1 \\
& \forall k: 1 \leq k-(t-1) \leq \mathrm{MD}_{i}^{b}, \forall i \in \Lambda_{b}, \forall b \in B, \forall t \\
& -o_{i(t-1)}^{b}+o_{i t}^{b}-u_{i t}^{b} \leq 0, \forall i \in \Lambda_{b}, \forall b \in B, \forall t \\
& o_{i(t-1)}^{b}-o_{i t}^{b}-v_{i t}^{b} \leq 0, \forall i \in \Lambda_{b}, \forall b \in B, \forall t \\
& o_{i t}^{b}, u_{i t}^{b}, v_{i t}^{b} \in\{0,1\}, \forall i \in \Lambda_{b}, \forall b \in B, \forall t
\end{aligned}
$$

where

$$
\begin{aligned}
& \mathcal{Q}(o, u, v, \xi)=\min \sum_{t=1}^{T} \sum_{b \in B} \sum_{i \in \Lambda_{b}} F_{c}\left(q_{i t}^{b}(\xi)\right) \\
& \quad+\sum_{t=1}^{T} \gamma_{t}^{+} Q_{t}^{I+}(\xi)+\sum_{t=1}^{T} \gamma_{t}^{-} Q_{t}^{I-}(\xi) \\
& L_{i}^{b} o_{i t}^{b} \leq q_{i t}^{b}(\xi) \leq U_{i}^{b} o_{i t}^{b}, \forall i \in \Lambda_{b}, \forall b \in B, \forall t \\
& q_{i t}^{b}(\xi)-q_{i(t-1)}^{b}(\xi) \leq\left(2-o_{i(t-1)}^{b}-o_{i t}^{b}\right) L_{i}^{b} \\
& \quad+\left(1+o_{i(t-1)}^{b}-o_{i t}^{b}\right) \mathrm{UR}_{i}^{b}, \forall i \in \Lambda_{b}, \forall b \in B, \forall t
\end{aligned}
$$

$$
\begin{aligned}
& q_{i(t-1)}^{b}(\xi)-q_{i t}^{b}(\xi) \leq\left(2-o_{i(t-1)}^{b}-o_{i t}^{b}\right) L_{i}^{b} \\
& \quad+\left(1-o_{i(t-1)}^{b}+o_{i t}^{b}\right) \mathrm{DR}_{i}^{b}, \forall i \in \Lambda_{b}, \forall b \in B, \forall t \\
& Q_{t}^{G}(\xi)=\sum_{b \in \mathrm{BG}} \sum_{i \in \Lambda_{b}} q_{i t}^{b}(\xi), \forall t \\
& Q_{t}^{W}(\xi)=\sum_{b \in \mathrm{BW}} \hat{q}_{t}^{b}(\xi), \forall t \\
& Q_{t}^{I+}(\xi)-Q_{t}^{I-}(\xi)=\sum_{b \in B} D_{b t}(\xi) \\
& \quad-\left(Q_{t}^{W}(\xi)+Q_{t}^{G}(\xi)\right), \forall t \\
& -C_{i j} \leq \sum_{b \in B} K_{i j}^{b}\left(\hat{q}_{t}^{b}(\xi)+\sum_{r \in \Lambda_{b}} q_{r t}^{b}(\xi)-D_{b t}(\xi)\right) \\
& \quad \leq C_{i j}, \forall(i, j) \in \mathcal{E}, \forall t \\
& \hat{q}_{t}^{b}(\xi) \leq W_{b t}(\xi), \forall b \in B, \forall t \\
& \operatorname{Pr}\left(-\Delta \leq Q_{t}^{W}(\xi)+Q_{t}^{G}(\xi)-\sum_{b \in B} D_{b t}(\xi) \leq \Delta, \forall t\right) \\
& \quad \geq 1-\epsilon, \\
& E\left[\sum_{t=1}^{T} Q_{t}^{W}(\xi)\right] \geq \beta E\left[\sum_{t=1}^{T} \sum_{b \in \mathrm{BW}} W_{b t}(\xi)\right] \\
& q_{i t}^{b}(\xi), \hat{q}_{t}^{b}(\xi), Q_{t}^{G}(\xi), Q_{t}^{W}(\xi), Q_{t}^{I \pm}(\xi) \geq 0 .
\end{aligned}
$$

In the above formulation, $F_{c}(\cdot)$ represents the fuel cost function. The objective function (1) is composed of the unit commitment costs in the first stage, and the fuel cost as well as the penalty cost due to load imbalance in the second stage. Minimum up-/down-time constraints (2) and (3) mean the status (on or off) of each unit should last for a minimum time once it is started up or shut down. Constraints (4) and (5) indicate the start-up and shut-down operations for each unit. Constraints (8) describe the upper and lower bounds of power output of each unit, and ramping constraints (9) and (10) limit the maximum increment or decrement of power generation of each unit between two adjacent periods. Constraints (11) and (12) represent the total thermal generation and the actual wind power utilized. Constraints (13) describe the possible load imbalance which is penalized in the objective function. Constraints (14) indicate the transmission capacity constraints and constraints (15) describe that the wind power utilized should be no more than the maximum available wind power. The chance constraint (16) requires that the chance of load imbalance beyond the tolerance level should be below a pre-defined risk level. Finally, the expected value constraint (17) can guarantee the usage of wind power output no less than a certain ratio of the maximum available wind power.

\section{Solution Methodology}

In this section, a combined SAA algorithm is developed to solve the expected value and chance constrained two-stage stochastic program. In this approach, an empirical distribution is used to approximate the actual distribution [30]-[32]. The combined SAA framework contains three parts: scenario generation, convergence analysis, and solution validation.

\section{A. Convergence Analysis}

The asymptotic consistency properties for expected value constrained stochastic program and for chance constrained sto- 
chastic program have been studied separately in [30] and [31], among others. These existing results can be applied to show the convergence property of the combined SAA algorithm for the model containing both expected value and chance constraints. First, an SAA problem of the original true problem can be obtained by reformulating (e.g., by taking samples) the expected value constraint only, without reformulating the chance constraint (denoted as SAA-EV). Second, the final combined SAA problem can be obtained by further reformulating the chance constraint (e.g., by taking samples) for the SAA-EV problem. It can be claimed that this combined SAA problem converges to the original true problem almost surely, i.e., the asymptotic consistency property holds for the combined SAA problem. The detailed proofs are shown in the Appendix.

\section{B. Scenario Generation}

Monte Carlo simulation is used to generate scenarios for the wind power output and load. Assuming the wind power output follows a multivariate normal distribution $N(\mu, \Sigma)$ [11] (note that this framework can also accommodate other distributions including Weibull distribution), where $\mu$ is the forecasted value of the wind power output and matrix $\Sigma$ denotes its forecast error, the Monte Carlo simulation is run to generate $N$ scenarios and each scenario has the same probability $1 / N$. Now the secondstage objective function can be replaced by

$$
\begin{aligned}
\frac{1}{N} \sum_{n=1}^{N}\left(\sum_{t=1}^{T} \sum_{b \in B} \sum_{i \in \Lambda_{b}} F_{c}\left(q_{i t}^{b}\left(\xi^{n}\right)\right)\right. & +\sum_{t=1}^{T} \gamma_{t}^{+} Q_{t}^{I+}\left(\xi^{n}\right) \\
& \left.+\sum_{t=1}^{T} \gamma_{t}^{-} Q_{t}^{I-}\left(\xi^{n}\right)\right) .
\end{aligned}
$$

For the expected value constraint, the Monte Carlo simulation is used to generate $N$ scenarios to estimate constraint (17) by

$$
\frac{1}{N} \sum_{n=1}^{N} \sum_{t=1}^{T} Q_{t}^{W}\left(\xi^{n}\right) \geq \frac{\beta}{N} \sum_{n=1}^{N} \sum_{t=1}^{T} \sum_{b \in B W} W_{b t}\left(\xi^{n}\right) .
$$

Meanwhile, the chance constraint (16) can be estimated as follows [32]:

$$
\begin{aligned}
\frac{1}{N} \sum_{n=1}^{N} \mathbf{1}_{[-\Delta, \Delta]}\left(Q_{t}^{W}\left(\xi^{n}\right)\right. & +Q_{t}^{G}\left(\xi^{n}\right) \\
& \left.-\sum_{b \in B} D_{b t}\left(\xi^{n}\right), \forall t\right) \geq 1-\epsilon
\end{aligned}
$$

where $\mathbf{1}_{[-\Delta, \Delta]}(\cdot)$ is an indicator function, i.e.,

$$
\mathbf{1}_{[-\Delta, \Delta]}(x)= \begin{cases}1, & \text { if } x \in[-\Delta, \Delta] \\ 0, & \text { if } x \notin[-\Delta, \Delta]\end{cases}
$$

Similarly, constraints (8)-(15) can be replaced respectively by scenario-based constraints. Then, the ultimate SAA problem is provided in the following Section III-B2 after linearizing constraint (21).

1) MILP Reformulation of Chance Constraint: After the scenarios are generated, the chance constraint is converted to an indicator function (21) in the SAA problem. It is still difficult to solve (21) directly since it is not convex. So an MILP model is used to reformulate this sampled chance constraint. For a given sample size $N$, binary decision variables $z$ are used: for each $n$ if $z_{n}=0$, then the chance constraint is satisfied in the corresponding scenario; otherwise, $z_{n}=1$. In addition, a big " $M$ " is introduced to model the case when the chance constraint is violated. Accordingly, the chance constraint is equivalent to limiting the number of $z_{n}, 1 \leq n \leq N$, to be ones. Then, the chance constraint can be represented as follows:

$$
\begin{aligned}
& -\Delta-M z_{n} \leq Q_{t}^{W}\left(\xi^{n}\right)+Q_{t}^{G}\left(\xi^{n}\right)-\sum_{b \in B} D_{b t}\left(\xi^{n}\right) \\
& \leq \Delta+M z_{n}, \quad \forall t, \forall n, \\
& \sum_{n=1}^{N} z_{n} \leq N \epsilon, z_{n} \in\{0,1\}, \quad \forall n .
\end{aligned}
$$

2) Reformulation of SAA Problem: The ultimate SAA problem can be described as follows:

$$
\begin{array}{ll} 
& \min \sum_{t=1}^{T} \sum_{b \in B} \sum_{i \in \Lambda_{b}}\left(\mathrm{SU}_{i}^{b} u_{i t}^{b}+\mathrm{SD}_{i}^{b} v_{i t}^{b}\right) \\
& +\frac{1}{N} \sum_{n=1}^{N}\left(\sum_{t=1}^{T} \sum_{b \in B} \sum_{i \in \Lambda_{b}} F_{c}\left(q_{i t}^{b}\left(\xi^{n}\right)\right)\right. \\
& \left.+\sum_{t=1}^{T} \gamma_{t}^{+} Q_{t}^{I+}\left(\xi^{n}\right)+\sum_{t=1}^{T} \gamma_{t}^{-} Q_{t}^{I-}\left(\xi^{n}\right)\right) \\
\text { s.t. } \quad(2)-(6), & (8)-(15),(18), \forall \xi \in\left\{\xi^{1}, \ldots, \xi^{N}\right\}, \\
& (20),(22),(23) .
\end{array}
$$

\section{Solution Validation}

The basic idea for the validation process is to apply statistical techniques to approximate the upper and lower bounds of the optimal objective value of the SAA problem. The optimality gap can be obtained through the validation process with a confidence level. For notation brevity, the mathematical model can be abstracted as follows:

$$
\begin{array}{ll} 
& \min c^{T} x+E[Q(x, \xi)] \\
\text { s.t. } & A x \leq b \\
& E[G(x, y(\xi), \xi)] \leq \ell \\
& \operatorname{Pr}(H(x, y(\xi), \xi) \geq 0) \leq \epsilon \\
& x \geq 0
\end{array}
$$

where

$$
\begin{array}{ll} 
& Q(x, \xi)=\min d(\xi)^{T} y(\xi) \\
\text { s.t. } & T(\xi) x+W y(\xi)=h(\xi), y(\xi) \geq 0 .
\end{array}
$$

In the above formulation, constraint (26) represents constraint (2)-(6), constraint (27) represents constraint (17), constraint (28) represents constraint (16), and constraint (31) represents constraints (8)-(15).

1) Upper Bound: First of all, the expected value $E[Q(x, \xi)]$ is assumed to be well-defined and finite valued for a given distribution of $\xi$. It is known that any feasible solution can provide an upper bound of the optimal objective value. Let $\left\{\xi^{1}, \xi^{2}, \ldots, \xi^{N}\right\}$ be a sample of size $N$ to generate an SAA problem, and $\bar{x}$ be the optimal first stage solution of the SAA problem. Note that the solution obtained by the SAA-based 
approach is not guaranteed feasible to the original problem, i.e., it is possible that $\bar{x}$ is infeasible to the original problem. To estimate the confidence level for $\bar{x}$ to be feasible (which leads to an upper bound accordingly), the proposed combined SAA approach defines $p(x):=\operatorname{Pr}\{H(x, y(\xi), \xi) \geq 0\}$ and $q(x):=E[G(x, y(\xi), \xi)]$, and uses the following two constraints in the SAA problem:

$$
\begin{aligned}
& p(x) \leq \tilde{\epsilon} \\
& q(x) \leq \tilde{\ell}
\end{aligned}
$$

where $\tilde{\epsilon} \leq \epsilon$ and $\tilde{\ell} \leq \ell$. Constraints (32) and (33) are equivalent to the chance constraint (28) and the expected value constraint (27) when $\tilde{\epsilon}=\epsilon$ and $\tilde{\ell}=\ell$. Note that if the sample size $N$ is large enough, when $\tilde{\epsilon} \leq \epsilon$ and $\tilde{\ell} \leq \ell$, the feasible solution of the SAA problem is more likely to be feasible to the original problem. Now, for a given size $N$, first based on the method described in [32], the $(1-\tau)$ confidence upper bound is constructed for the chance constraint (28):

$$
U_{c}(\bar{x})=p_{N}(\bar{x})+z_{\tau} \sqrt{\frac{p_{N}(\bar{x})\left(1-p_{N}(\bar{x})\right)}{N}}
$$

where $p_{N}(\bar{x})=(1) /(N) \sum_{n=1}^{N} \mathbf{1}_{[0, \infty)}\left(H\left(\bar{x}, y\left(\xi^{n}\right), \xi^{n}\right)\right)$ and $z_{\tau}=\Phi^{-1}(1-\tau)$ is the $(1-\tau)$-quantile of the standard normal distribution. If $U_{c}(\bar{x})$ is less than or equal to the risk level $\epsilon$, then $\bar{x}$ is feasible for the chance constraint with the confidence level $(1-\tau)$. If not, the value of $\tilde{\epsilon}$ is decreased and the problem is solved again to check whether the updated optimal solution leads to a new value $U_{c}(\bar{x})$ less than or equal to $\epsilon$.

Based on the first step, i.e., $\bar{x}$ is feasible to constraint (32), the $(1-\tau)$ confidence upper bound is computed for the expected value constraint (cf. [30]):

$$
U_{e}(\bar{x})=q_{N}(\bar{x})+z_{\tau} \sqrt{\frac{\sum_{n=1}^{N}\left[G\left(\bar{x}, y\left(\xi^{n}\right), \xi^{n}\right)-q_{N}(\bar{x})\right]^{2}}{N(N-1)}}
$$

where $q_{N}(\bar{x})=(1) /(N) \sum_{n=1}^{N} G\left(\bar{x}, y\left(\xi^{n}\right), \xi^{n}\right)$. If $U_{e}(\bar{x})$ is less than or equal to $\ell, \bar{x}$ is feasible for the expected value constraint with the confidence level $(1-\tau)$. If not, the value of $\tilde{\ell}$ can be decreased to improve the chance of obtaining a feasible solution.

Since it is well-known that $\operatorname{Pr}(A \cap B)=\operatorname{Pr}(A) \operatorname{Pr}(B \mid A)$, $\bar{x}$ is feasible for the original problem with a confidence level $(1-\tau)^{2}$. The corresponding upper bound of the true problem is given as follows:

$$
U(\bar{x})=c^{T} \bar{x}+\frac{1}{N} \sum_{n=1}^{N} Q\left(\bar{x}, \xi^{n}\right)
$$

2) Lower Bound: The following Lagrange relaxation formulation (denoted as $P_{0}$ ) of the original problem is considered to obtain a lower bound:

$$
\min \left\{c^{T} x+E[Q(x, \xi)]+\pi(E[G(x, y(\xi), \xi)]-\ell)\right\}
$$

s.t. Constraints (26), (28), and (29).
Denote the optimal objective value of $P_{0}$ as $v_{0}$, and the optimal objective value of the original problem as $v^{*}$. From Lagrangian duality:

$$
v_{0} \leq v^{*}
$$

for any $\pi \geq 0$. Especially, the equation holds when $\pi$ is the optimal Lagrange multiplier and there is no duality gap.

Now, by generating $N$ scenarios, the chance constraint (28) is estimated as $(1) /(N) \sum_{n=1}^{N} \mathbf{1}_{[0, \infty)}\left(H\left(x, y\left(\xi^{n}\right), \xi^{n}\right)\right) \leq \epsilon$. The Lagrange relaxation problem can be approximated as follows (denoted as $\left.P_{1}\right)$ :

$$
\min c^{T} x+E[Q(x, \xi)]+\pi(E[G(x, y(\xi), \xi)]-\ell)
$$

s.t. Constraints (26) and (29)

$$
\frac{1}{N} \sum_{n=1}^{N} \mathbf{1}_{[0, \infty)}\left(H\left(x, y\left(\xi^{n}\right), \xi^{n}\right)\right) \leq \epsilon .
$$

Then, after the scenarios are generated (e.g., $N$ scenarios), the objective value in $P_{1}$ can be estimated by

$$
c^{T} x+\frac{1}{N} \sum_{n=1}^{N}\left(Q\left(x, \xi^{n}\right)+\pi\left(G\left(x, y\left(\xi^{n}\right), \xi^{n}\right)-\ell\right)\right)
$$

Finally, the Lagrange relaxation problem can be approximated as the following SAA problem (denoted as $P_{2}$ ):

$$
\begin{array}{r}
\min \left\{c^{T} x+\frac{1}{N} \sum_{n=1}^{N}\left(Q\left(x, \xi^{n}\right)\right.\right. \\
\left.\left.+\pi\left(G\left(x, y\left(\xi^{n}\right), \xi^{n}\right)-\ell\right)\right)\right\}
\end{array}
$$

s.t. Constraints (26) and (29)

$$
\frac{1}{N} \sum_{n=1}^{N} \mathbf{1}_{[0, \infty)}\left(H\left(x, y\left(\xi^{n}\right), \xi^{n}\right)\right) \leq \epsilon
$$

The SAA problem is solved with $N$ scenarios $M$ times. For these $M$ runs, for analysis convenience, the optimal values of $P_{1}$ are denoted as $\hat{v}_{1}^{P_{1}}, \hat{v}_{2}^{P_{1}}, \ldots, \hat{v}_{M}^{P_{1}}$, and the optimal values of $P_{2}$ as $\hat{v}_{1}^{P_{2}}, \hat{v}_{2}^{P_{2}}, \ldots, \hat{v}_{M}^{P_{2}}$. If $\hat{v}_{i}^{P_{1}}, i=1, \ldots, M$ are obtained, then the same scheme, as the one described in [32], can be followed, e.g., letting $L$ be the largest integer such that $B(L-$ $\left.1 ; \theta_{N}, M\right) \leq \tau$, where $\theta_{N}=B(\lfloor\epsilon N\rfloor ; \epsilon, N)$ with $B(k ; q, N)=$ $\sum_{i=0}^{k}\left(\begin{array}{c}N \\ i\end{array}\right) q^{i}(1-q)^{N-i}$ denoting the cdf of binomial distribution, and picking the $L$ th smallest optimal value of $P_{1}$ (denoted as $\left.\hat{v}_{L}^{P_{1}}\right)$. Based on the conclusions obtained in [32]:

$$
\operatorname{Pr}\left(\hat{v}_{L}^{P_{1}} \leq v_{0}\right)=1-\tau
$$

But, it is not straightforward to obtain $\hat{v}_{L}^{P_{1}}$. Fortunately, for each run $i=1,2, \ldots, M$, to solve $P_{2}, E\left[\hat{v}_{i}^{P_{2}}\right] \leq \hat{v}_{i}^{P_{1}}$ based on the inequality $E\left[\min _{x} f(x, \xi)\right] \leq \min _{x} E[f(x, \xi)]$ [33]. Thus, accordingly

$$
E\left[\hat{v}_{L}^{P_{2}}\right] \leq \hat{v}_{L}^{P_{1}}
$$


Now $E\left[\hat{v}_{L}^{P_{2}}\right]$ can be approximated $\}$ by statistical techniques [30]. Let $S$ denote the number of total iterations. In each iteration $s, 1 \leq s \leq S$, the SAA is solved with $N$ scenarios $M$ times to obtain $\hat{v}_{L}^{P_{2}}$, which is re-denoted as $\bar{v}_{L_{s}}^{P_{2}}$. Let

$$
\begin{aligned}
\bar{v}_{L} & =\frac{\bar{v}_{L_{1}}^{P_{2}}+\cdots+\bar{v}_{L_{S}}^{P_{2}}}{S} \\
\tilde{v} & =\bar{v}_{L}-z_{\tau} \sqrt{\frac{\sum_{s=1}^{S}\left[\bar{v}_{L_{s}}^{P_{2}}-\bar{v}_{L}\right]^{2}}{S(S-1)} .}
\end{aligned}
$$

It can be obtained that

$$
\operatorname{Pr}\left(\tilde{v} \leq E\left[\hat{v}_{L}^{P_{2}}\right]\right)=1-\tau .
$$

From inequalities (38), (41), (42) and (45), $\operatorname{Pr}\left(\tilde{v} \leq v^{*}\right) \geq(1-$ $\tau)^{2}$, i.e., $\tilde{v}$ is the lower bound of $v^{*}$ with a confidence level at least $(1-\tau)^{2}$.

\section{Summary of the Combined SAA Algorithm}

The combined SAA algorithm is summarized as follows (the flow chart is shown in Fig. 1):

Step 1) For $s=1,2, \ldots, S$, repeat the following steps:

1) For $m=1,2, \ldots, M$, repeat the following steps:

a) Set $\delta_{c}>0, \delta_{e}>0, \tau \in(0,1), \tilde{\epsilon}=\epsilon$, and $\tilde{\ell}=\ell$.

b) Solve the SAA problem with $N$ scenarios:

$\min \hat{f}_{N}(x)=c^{T} x+\frac{1}{N} \sum_{n=1}^{N} Q\left(x, \xi^{n}\right)$

s.t. $\quad A x \leq b, x \geq 0$

$\frac{1}{N} \sum_{n=1}^{N} \mathbf{1}_{(0, \infty)}\left(H\left(x, y\left(\xi^{n}\right), \xi^{n}\right)\right) \leq \tilde{\epsilon}$

$\frac{1}{N} \sum_{n=1}^{N} G\left(x, y\left(\xi^{n}\right), \xi^{n}\right) \leq \tilde{\ell}$

where the second stage is the same as (30) and (31). Let $\left(\bar{x}^{m}, \bar{y}^{m}\right)$ be the optimal solution to the SAA problem, and $\bar{\pi}^{m}$ be the optimal Lagrange multiplier.

c) Generate scenarios $\xi^{1}, \xi^{2}, \ldots, \xi^{N^{\prime}}$ for a large number $N^{\prime}$ and use (34) and (35) to obtain $U_{c}\left(\bar{x}^{m}\right)$ and $U_{e}\left(\bar{x}^{m}\right)$. Check whether $U_{c}\left(\bar{x}^{m}\right) \leq \epsilon$ and $U_{e}\left(\bar{x}^{m}\right) \leq \ell$ hold. If the former fails, reduce $\tilde{\epsilon}$ by $\delta_{c}$. If the latter fails, reduce $\tilde{\ell}$ by $\delta_{e}$. Return to b).

d) Estimate the corresponding upper bound using (36), based on the $N^{\prime}$ scenarios generated in c.

2) Pick the smallest upper bound in 1) as the approximated upper bound $\hat{g}^{s}$.

3) By using the Lagrange multiplier obtained in b), sort the $M$ optimal values of $P_{2}$ in a nondecreasing order, e.g., $\hat{v}_{1}^{P_{2}} \leq \hat{v}_{2}^{P_{2}} \leq \cdots \hat{v}_{M}^{P_{2}}$. Pick the $L$ th smallest optimal value $\hat{v}_{L}^{\bar{P}_{2}}$ and denote it as $\bar{v}_{L_{s}}^{P_{2}}$.

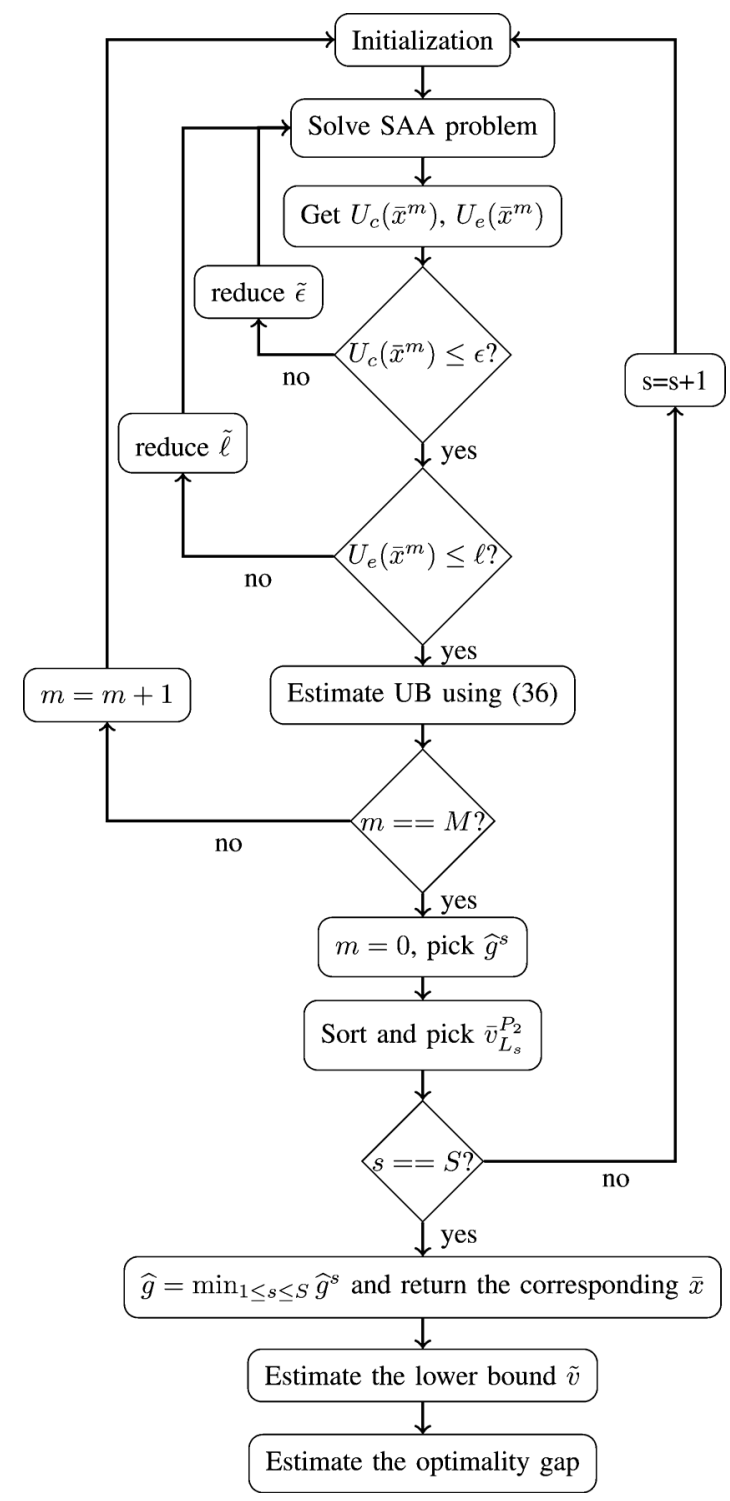

Fig. 1. Proposed Combined SAA Algorithm.

Step 2) Take the minimum of $\hat{g}^{1}, \hat{g}^{2}, \ldots, \hat{g}^{S}$ to get the upper bound $\hat{g}=\min _{1 \leq s \leq S} \hat{g}^{s}$ and return the corresponding $\bar{x}$.

Step 3) Compute $\bar{v}_{L}$ based on (43) and the estimated lower bound $\tilde{v}$ based on (44).

Step 4) Estimate the optimality gap using $(\hat{g}-\tilde{v}) / \tilde{v} \times 100 \%$ with the confidence level at least $(1-\tau)^{2}$.

\section{Computational Results}

In this section, case studies for a six-bus and two revised IEEE 118-bus systems are performed to show the effectiveness of the proposed approach. A sensitivity analysis of the SAA problem is first performed on the six-bus system. Then the combined SAA algorithm described in Section III is applied to solve the two revised IEEE-118 bus systems. The time horizon is 24 hours at 1 hour time step. C++ with CPLEX 12.1 is used to implement the proposed formulations and algorithms. All the experiments are conducted on a computer workstation with 4 Intel Cores and 8 GB RAM. 
TABLE I

SiX-Bus SySTEM With DifFERENT UTILIZATION LEVELS

\begin{tabular}{|c|c|c|}
\hline Utilization $\beta$ & Obj. (\$) & CPU Time (sec) \\
\hline $60 \%$ & 26977.5 & 2.7 \\
$70 \%$ & 27646.2 & 20.5 \\
$80 \%$ & 32991.9 & 10.2 \\
$85 \%$ & 39603.9 & 9.5 \\
$90 \%$ & 46215.9 & 9.1 \\
\hline
\end{tabular}

TABLE II

SiX-Bus System With DifFERENT RISK LEVELS

\begin{tabular}{|c|c|c|}
\hline Risk Level $\epsilon$ & Obj. (\$) & CPU Time (sec) \\
\hline 0.05 & 27235.5 & 77.9 \\
0.10 & 26649.0 & 161.4 \\
0.15 & 26284.3 & 160.2 \\
0.20 & 25928.5 & 821.6 \\
\hline
\end{tabular}

\section{A. Six-Bus System}

The six-bus system contains three thermal generators and one wind farm. The settings of the wind farm, the thermal generators, the load forecasts, and the transmission lines are the same as the ones described in [19], except the wind farm located at bus $B_{2}$.

The proposed approach follows [11] to generate wind power output scenarios subject to the multi-variate normal distribution. The wind power forecast is based on the hourly forecasted wind power provided in Table IV in [11]. And wind forecast errors are assumed to be $10 \%$ deviations of the forecasted values in each time period. The percentage of wind power output over load for the six-bus system is $P_{w / d}^{\text {six }}=24 \%$. This percentage is equal to the total forecasted wind power output (MWh) divided by the total load (MWh), over the 24-hour time horizon.

Sensitivity analyses are provided on the utilization rate in the expected value of wind power output constraint, which is used to decide the overall wind power utilization, and the risk level in the chance constraint, which aims at controlling the system balance and avoiding load curtailment.

The sample size $N$ is set to be 50 and the unit imbalance cost $\gamma_{t}^{+}=\gamma_{t}^{-}=\$ 30 / \mathrm{MWh}$. For the derived reformulation of chance constraint (21) in the SAA problem, $\epsilon$ is set to be zero. That is, the surplus or shortage of power supply is assumed within a tolerance level $\Delta$ for all the sampled scenarios, i.e., $z_{n}=0$ in constraint (22) for all $n$. The computational results are reported in Table I. It can be observed that the total cost (column "obj." in Table I) increases as the utilization rate increases from $60 \%$ to $90 \%$. This is because the utilization policy becomes more restrictive as $\beta$ increases. Accordingly, more uncertainties have to be accommodated by other thermal units in the power grid. For example, some thermal units have to be online to provide sufficient ramping capabilities to cover some extreme scenarios. And sometimes thermal units have to yield to wind power generation due to the transmission congestion.

To investigate the significance of the settings on the chance constraint, the results are reported in Table II with different risk levels. The sample size is set to be 50 and $\beta$ is set to be $70 \%$.

It can be observed that the total cost decreases as the risk level increases from 0.05 to 0.2 , because the load imbalance policy becomes less restrictive as the risk level increases.
TABLE III

118-Bus SYSTEM WiTH DifFERENT COMBINATIONS OF ITERATIONS AND SAMPLE SIZES

\begin{tabular}{cccccc}
\hline$\left(S \times M, N^{\prime}\right)$ & $\mathrm{N}$ & LB & UB & Gap & Time (sec) \\
\hline \multirow{3}{*}{$(5 \times 5,100)$} & 10 & 585578 & 588178 & $0.44 \%$ & 127 \\
& 20 & 585873 & 588178 & $0.39 \%$ & 402 \\
& 50 & 586651 & 588206 & $0.27 \%$ & 1379 \\
\hline \hline \multirow{3}{*}{$(10 \times 5,500)$} & 10 & 586133 & 587700 & $0.27 \%$ & 316 \\
& 20 & 586818 & 587700 & $0.15 \%$ & 783 \\
\hline \hline & 50 & 587144 & 587734 & $0.10 \%$ & 2319 \\
\hline \multirow{2}{*}{$(20 \times 10,1000)$} & 10 & 586829 & 587758 & $0.15 \%$ & 1364 \\
& 20 & 587219 & 587758 & $0.09 \%$ & 3325 \\
& 50 & 587380 & 587793 & $0.07 \%$ & 9005 \\
\hline
\end{tabular}

Finally, the experiments are run with different scenario sizes and we observe that the optimal objective values converge as the sample size increases to 70 (the risk level $\epsilon$ is set to be 0.1 and utilization level $\beta$ is set to be $70 \%$ ).

\section{B. Revised 118-Bus Systems}

A revised IEEE 118-bus systems (named 118SW) based on the one given online at http://motor.ece.iit.edu/data is studied in this section. Apart from the 33 thermal generators in the original IEEE 118 -bus system, $118 \mathrm{SW}$ is created by adding a single centralized wind farm. In the $118 \mathrm{SW}$ system, the wind power output is scaled based on the ones described in the six-bus system and the percentage of wind power output over load is $P_{w / d}^{118 \mathrm{SW}}=21 \%$.

The combined SAA algorithm is implemented for $118 \mathrm{SW}$ and the computational results are reported in Table III. The first column indicates that different combinations of validation settings [i.e., iteration numbers $(S, M)$ and validation scenario numbers $\left.\left(N^{\prime}\right)\right]$ are considered in the experiments. The scenario size of the SAA problem is given in the second column. The lower bound (LB) and upper bound (UB) obtained by the SAA algorithm are reported in the third and fourth columns. The fifth column represents the gap which is calculated by $(\mathrm{UB}-\mathrm{LB}) /(\mathrm{LB}) \times 100 \%$. The sixth column reports the CPU time of the algorithm. In the experiment, $z_{\tau}$ is set to be 1.2 (i.e., $\tau=11.5 \%$ ) in the combined SAA algorithm. So the confidence level of the reported LB/UB is at least $(1-\tau)^{2}=78.3 \%$.

From the table, it can be observed that: First, when the scenario size of the SAA problem is $N=10$, a small optimality gap $0.44 \%$ is already obtained. Such an observation suggests that the solution of the SAA problem with the scenario size $N=10$ is already very close to the solution of the true problem. Second, the optimality gap decreases as the scenario sizes of the SAA problem and the validation problem increase. In the last row, when the scenario size of the SAA problem is $N=$ 50 with the iteration number and the validation scenario size being $(20 \times 10,1000)$, the smallest optimality gap is achieved at $0.07 \%$. In addition, the sample variances of the SAA optimal objective values with different scenario sizes for the setting $(20 \times 10,1000)$ are, in the form (scenario size, standard deviation/mean), $(10,0.22 \%),(20,0.16 \%)$, and $(50,0.09 \%)$. It can be observed that the standard deviations decrease as well as the sample size increases. Therefore, the solution quality is improved when larger scenario sizes are taken to solve the problem. Finally, it can be observed that the trade-off for increased accuracy is longer computation time. For instance, the 


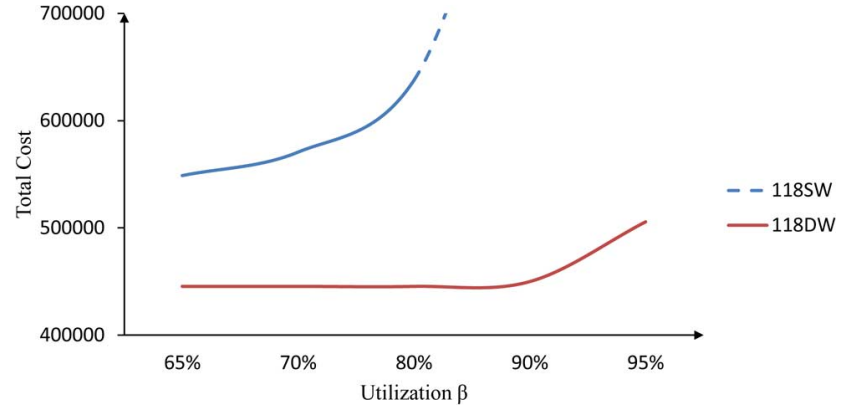

Fig. 2. Cross comparison of $118 \mathrm{SW}$ and $118 \mathrm{DW}$.

computation time increases from 127 seconds to 9005 seconds when the gap decreases from $0.44 \%$ to $0.07 \%$.

\section{Distributed Wind Power System 118DW}

Furthermore, a distributed wind power system 118DW is created by adding multiple wind farms at different buses in the IEEE-118 bus system.

1) Cross Comparison of $118 \mathrm{SW}$ and $118 \mathrm{DW}$ Systems: In this comparison, the 118DW system contains three distributed wind farms. The wind power output for each follows an identical normal distribution. The total wind power outputs are adjusted to be the same for both systems. The percentage of wind power output over load $P_{w / d}$ is adjusted to be $40 \%$ for both systems. The results are summarized in Fig. 2.

It can be observed that the total cost for the $118 \mathrm{DW}$ system has remained the same until the utilization level reaches $\beta=$ $90 \%$. The reason behind this is that the $118 \mathrm{DW}$ system utilizes a high portion of wind power even without the utilization constraint. But an utilization rate higher than $95 \%$ incurs a higher system cost. On the other hand, the $118 \mathrm{SW}$ system utilizes a relative low portion of wind power, because large wind power generation from a centralized bus causes transmission congestions. Enforcing the proposed expected value constraint can help improve the wind power utilization. However, the system becomes infeasible (dotted line) when the utilization rate is larger than $82 \%$, because the transmission capacity limits are reached. Due to the distributed wind resources, the 118DW system can take advantage of transmission capacities at different locations. So the 118DW system in nature has higher utilization of wind power over the $118 \mathrm{SW}$ system. And enforcing expected value constraint on wind power utilization has a smaller impact on the total system cost of the 118DW system than that of the $118 \mathrm{SW}$ system.

2) Impact of Expected Value Constraint on 118DW: The experiments are run on 118DW in this section and the impact of the expected value constraint on distributed wind power resources is analyzed. In total, there are 10 wind farms at 10 different buses (bus numbers are shown in Table IV). The wind power output is scaled and $P_{w / d}^{118} \mathrm{DW}=49 \%$ in this section. Under such a setting, the associated wind power uncertainties affect the power grid operations dramatically.

First, the expected value constraint for wind power utilization is relaxed and then the stochastic optimization problem is solved. The utilization rates of wind power at specific buses are summarized in Table IV. The lowest utilization and highest utilization are observed in $B_{36}$ and $B_{96}$, respectively. The reason
TABLE IV

118 DW-Without EXPECTED VALUE CONSTRAINT

\begin{tabular}{|c|c||c|c|}
\hline Bus NO. & Utilization & Bus NO. & Utilization \\
\hline$B_{6}$ & $90 \%$ & $B_{56}$ & $65 \%$ \\
$B_{16}$ & $78 \%$ & $B_{66}$ & $76 \%$ \\
$B_{26}$ & $73 \%$ & $B_{76}$ & $74 \%$ \\
$B_{36}$ & $40 \%$ & $B_{86}$ & $71 \%$ \\
$B_{46}$ & $90 \%$ & $B_{96}$ & $96 \%$ \\
\hline
\end{tabular}

TABLE V

118DW-With EXPeCted VAlue Constraint

\begin{tabular}{|c|c||c|c|}
\hline Bus NO. & Utilization & Bus NO. & Utilization \\
\hline$B_{6}$ & $97 \%$ & $B_{56}$ & $69 \%$ \\
$B_{16}$ & $84 \%$ & $B_{66}$ & $90 \%$ \\
$B_{26}$ & $95 \%$ & $B_{76}$ & $85 \%$ \\
$B_{36}$ & $55 \%$ & $B_{86}$ & $78 \%$ \\
$B_{46}$ & $93 \%$ & $B_{96}$ & $98 \%$ \\
\hline
\end{tabular}

of low utilization is that there is one thermal generator in $B_{36}$ and the capacity of the transmission line connected with this bus is relatively lower compared with others. The wind power has to be curtailed due to the limited transmission line capacity. Conversely, the total capacity of the transmission lines connected with $B_{96}$ is much larger (there are five transmission lines connected with $B_{96}$ ) and no generator is located there.

Then the problem with the expected value constraint for wind power utilization enforced is solved. The utilization rate is set to be $85 \%$. The corresponding utilization rates at each studied bus are reported in Table V. It can be observed that the utilization rates at all buses are increased compared with those in Table IV. On the other hand, it can be observed that the utilization increments at different buses are different as the largest increment is $22 \%\left(B_{26}\right)$ and the smallest increment is $2 \%\left(B_{96}\right)$, which shows the advantage of applying the proposed solution approach to minimize the total cost while ensuring the overall high wind power utilization rate. In other words, the model can help coordinate generation injections at different buses and provide the optimal generation strategy at each bus. Finally, it is worth noting that there is a trade-off to enforce a higher utilization of wind power. Imposing the utilization constraint makes the thermal units and transmission lines accommodate wind power uncertainties. The trade-off of achieving such a high utilization is to incur $4.4 \%$ increment of the total system cost in this case.

\section{CONCLUSION}

In this paper, an expected value constrained stochastic program is proposed to study the uncertain wind power generation. By adjusting the utilization rate in the expected value constraint, a system operator can change the overall usage level of wind resources. In addition, a chance constraint is applied to restrict the load imbalance with a small probability. Thus, this model incorporates both the expected value and chance constraints. Furthermore, an innovative combined SAA algorithm is developed to solve the problem. The sensitivity analysis is also performed on different risk levels and wind utilization rates for the six-bus system. Finally, the computational results of the revised IEEE 
118-bus systems exhibit the scalability of the proposed algorithm. From the experiments, it can be observed that the proposed approach has the potential to help enhance the unit commitment procedure by ISOs/RTOs to ensure the utilization of wind power, while maintaining system reliability. It is worthwhile to note that by the previous study [19], ISOs can address a high wind power utilization with a chance-constrained stochastic program. The expected value constraint, from another perspective, allows ISOs to enforce an overall high wind power usage. ISOs can accordingly choose different policies and corresponding constraints to enhance the wind power utilization level. In some extreme cases, ISOs might want to have both chance and expected value constraints on the wind power utilization. The proposed combined SAA algorithm in this research can also be applied to solve these cases.

\section{APPENDIX}

In this Appendix, we prove that the asymptotic consistency property holds for the combined SAA problem. We first state the following theorem (described in [34]), which will be used later on in our proof.

Theorem 1: [Zorich 2004] Let $\left\{f_{n}: n \in \mathbb{N}\right\}$ be a family of functions $f_{n}: \Lambda \mapsto \mathbb{C}$ and $f: \Lambda \mapsto \mathbb{C}$. Let $\Lambda^{\prime}$ be the set of limit points of $\Lambda$ and $\lambda_{\infty} \in \Lambda^{\prime}$. Assume that

1) $\lim _{n \rightarrow \infty} f_{n}(\lambda)=f(\lambda)$ uniformly on $\Lambda$ and

2) $\lim _{\lambda \rightarrow \lambda_{\infty}} f_{n}(\lambda)=A_{n}$ exists.

Then

1) $\lim _{n \rightarrow \infty} A_{n}=A$ exists and

2) $\lim _{\lambda \rightarrow \lambda_{\infty}} f(\lambda)=A$. That is, $\lim _{\lambda \rightarrow \lambda_{\infty}} \lim _{n \rightarrow \infty} f_{n}(\lambda)=$ $\lim _{n \rightarrow \infty} \lim _{\lambda \rightarrow \lambda_{\infty}} f_{n}(\lambda)$.

Now we are ready to describe and prove our claim.

Theorem 2: The combined SAA problem converges to the original true problem, i.e., the asymptotic consistency property holds for the combined SAA problem.

Proof: Let set $\Lambda=\{1,1 / 2,1 / 3, \ldots, 1 / m, \ldots\}$. Now we let $\lambda_{m}=1 / m$ and define

$$
f_{n}\left(\lambda_{m}\right)=\min c^{T} x+E[Q(x, \xi)]
$$

s.t. $A x \leq b$,

Chance constraint reformulation with $n$ samples

Expected value constraint reformulation with $m$ samples

which is essentially the combined SAA problem.

We also define

$$
f\left(\lambda_{m}\right)=\min c^{T} x+E[Q(x, \xi)]
$$

s.t. $A x \leq b$,

Original chance constraint

Expected value constraint reformulation with $m$ samples.

Meanwhile, we notice that the limit point for $\Lambda$ corresponds to the case $m=\infty$. Then for the point $\lambda_{\infty} \in \Lambda^{\prime}$ with $\Lambda^{\prime}$ be the set of limit points of $\Lambda$, we have

$$
\begin{array}{ll} 
& f_{n}\left(\lambda_{\infty}\right)=\min c^{T} x+E[Q(x, \xi)] \\
\text { s.t. } & A x \leq b \\
& \text { Chance constraint reformulation with } n \text { samples } \\
& \text { Original expected value constraint }
\end{array}
$$

and $f\left(\lambda_{\infty}\right)$ represents the objective value of the original problem. According to [31, Proposition 2.2], we have $\lim _{n \rightarrow \infty} f_{n}\left(\lambda_{m}\right)=f\left(\lambda_{m}\right)$ uniformly on $\Lambda$, and according to [30, Proposition 1], we have that for each $n \in \mathbb{N}$, $\lim _{\lambda_{m} \rightarrow \lambda_{\infty}} f_{n}\left(\lambda_{m}\right)=A_{n}$ exists. Therefore, according to Theorem 1, we have $\lim _{\lambda_{m} \rightarrow \lambda_{\infty}} \lim _{n \rightarrow \infty} f_{n}\left(\lambda_{m}\right)=$ $\lim _{n \rightarrow \infty} \lim _{\lambda_{m} \rightarrow \lambda_{\infty}} f_{n}\left(\lambda_{m}\right)$. In other words, the combined SAA problem converges to the original true problem almost surely, regardless of the order of taking limits.

\section{ACKNOWLEDGMENT}

The authors would like to thank the editor and four reviewers for their sincere suggestions on improving the quality of this paper.

\section{REFERENCES}

[1] H. Holttinen, P. Meibom, A. Orths, B. Lange, M. O’Malley, J. O. Tande, A. Estanqueiro, E. Gomez, L. Söder, G. Strbac, J. C. Smith, and F. van Hulle, "Impacts of large amounts of wind power on design and operation of power systems, results of IEA collaboration," Wind Energy, vol. 14, no. 2, pp. 179-192, 2011.

[2] US DOE, " $20 \%$ wind energy by 2030 : Increasing wind energy's contribution to U.S. electricity supply," . Washington, DC, 2008.

[3] A. J. Wood and B. F. Wollenberg, Power Generation, Operation and Control, 2nd ed. New York, NY, USA: Wiley, 1996.

[4] R. Doherty and M. O'Malley, “A new approach to quantify reserve demand in systems with significant installed wind capacity," IEEE Trans. Power Syst., vol. 20, no. 2, pp. 587-595, May 2005.

[5] M. A. Ortega-Vazquez and D. S. Kirschen, "Estimating the spinning reserve requirements in systems with significant wind power generation penetration," IEEE Trans. Power Syst., vol. 24, no. 1, pp. 114-124, Feb. 2009

[6] L. Wu, M. Shahidehpour, and T. Li, "Stochastic security-constrained unit commitment," IEEE Trans. Power Syst., vol. 22, no. 2, pp. 800-811, May 2007.

[7] Q. Wang, J. Wang, and Y. Guan, "Stochastic unit commitment with uncertain demand response," IEEE Trans. Power Syst., vol. 28, no. 1, pp. 562-563, Feb. 2013.

[8] P. Ruiz, C. Philbrick, E. Zak, K. Cheung, and P. Sauer, "Uncertainty management in the unit commitment problem," IEEE Trans. Power Syst., vol. 24, no. 2, pp. 642-651, May 2009

[9] A. Tuohy, P. Meibom, E. Denny, and M. O'Malley, "Unit commitment for systems with significant wind penetration," IEEE Trans. Power Syst., vol. 24, no. 2, pp. 592-601, May 2009.

[10] Q. Wang, J. Wang, and Y. Guan, "Price-based unit commitment with wind power utilization constraints," IEEE Trans. Power Syst., vol. 28, no. 3, pp. 2718-2726, Aug. 2013.

[11] J. Wang, M. Shahidehpour, and Z. Li, "Security-constrained unit commitment with volatile wind power generation," IEEE Trans. Power Syst., vol. 23, no. 3, pp. 1319-1327, Aug. 2008.

[12] V. S. Pappala, I. Erlich, K. Rohrig, and J. Dobschinski, "A stochastic model for the optimal operation of a wind-thermal power system," IEEE Trans. Power Syst., vol. 24, no. 2, pp. 940-950, May 2009.

[13] E. M. Constantinescu, V. M. Zavala, M. Rocklin, S. Lee, and M. Anitescu, "A computational framework for uncertainty quantification and stochastic optimization in unit commitment with wind power generation," IEEE Trans. Power Syst., vol. 26, no. 1, pp. 431-441, Feb. 2011.

[14] L. Wu, M. Shahidehpour, and Z. Li, "Comparison of scenario-based and interval optimization approaches to stochastic SCUC," IEEE Trans. Power Syst., vol. 27, no. 2, pp. 913-921, May 2012.

[15] J. Rogers, S. Fink, and K. Porter, Examples of Wind Energy Curtailment Practices, NREL, , Subcontract Report NREL/SR-550-48737, 2010.

[16] J. R. Birge and F. Louveaux, Introduction to Stochastic Programming. New York, NY, USA: Springer-Verlag, 1997.

[17] U. A. Ozturk, M. Mazumdar, and B. A. Norman, "A solution to the stochastic unit commitment problem using chance constrained programming," IEEE Trans. Power Syst., vol. 19, no. 3, pp. 1589-1598, Aug. 2004.

[18] H. Yu, C. Y. Chung, K. P. Wong, and J. H. Zhang, "A chance constrained transmission network expansion planning method with consideration of load and wind farm uncertainties," IEEE Trans. Power Syst., vol. 24, no. 3, pp. 1568-1576, Aug. 2009. 
[19] Q. Wang, Y. Guan, and J. Wang, "A chance-constrained two-stage stochastic program for unit commitment with uncertain wind power output," IEEE Trans. Power Syst., vol. 27, no. 1, pp. 206-215, Feb. 2012.

[20] T. Li, M. Shahidehpour, and Z. Li, "Risk-constrained bidding strategy with stochastic unit commitment," IEEE Trans. Power Syst., vol. 22, no. 1 , pp. 449-458, Feb. 2007.

[21] L. Wu, M. Shahidehpour, and Z. Li, "Genco's risk-constrained hydrothermal scheduling," IEEE Trans. Power Syst., vol. 23, no. 4, pp. 1847-1858, Nov. 2008.

[22] L. V. L. Abreu, M. E. Khodayar, M. Shahidehpour, and L. Wu, "Riskconstrained coordination of cascaded hydro units with variable wind power generation," IEEE Trans. Sustain. Energy, to be published.

[23] R. Jiang, M. Zhang, G. Li, and Y. Guan, "Two-stage network constrained robust unit commitment problem," Eur. J. Oper. Res., vol. 234, pp. 751-762, 2014.

[24] L. Baringo and A. Conejo, "Offering strategy via robust optimization," IEEE Trans. Power Syst., vol. 26, no. 3, pp. 1418-1425, Aug. 2011.

[25] R. Jiang, J. Wang, and Y. Guan, "Robust unit commitment with wind power and pumped storage hydro," IEEE Trans. Power Syst., vol. 27, no. 2, pp. 800-810, May 2012.

[26] C. Zhao and Y. Guan, "Unified stochastic and robust unit commitment," IEEE Trans. Power Syst., vol. 28, no. 3, pp. 3353-3361, Aug. 2013.

[27] R. Rockafellar and S. Uryasev, "Optimization of conditional value-atrisk," J. Risk, vol. 2, pp. 21-42, 2000.

[28] A. Botterud, Z. Zhou, J. Wang, R. Bessa, H. Keko, J. Sumaili, and V. Miranda, "Wind power trading under uncertainty in LMP markets," IEEE Trans. Power Syst., vol. 27, no. 2, pp. 894-903, May 2012.

[29] R. A. Jabr, "Robust self-scheduling under price uncertainty using conditional value-at-risk," IEEE Trans. Power Syst., vol. 20, no. 4, pp. 1852-1858, Nov. 2005.

[30] W. Wang and S. Ahmed, "Sample average approximation of expected value constrained stochastic programs," Oper. Res. Lett., vol. 36, no. 5, pp. 515-519, 2008.

[31] B. K. Pagnoncelli, S. Ahmed, and A. Shapiro, "Sample average approximation method for chance constrained programming: Theory and applications," J. Optimiz. Theory Applicat., vol. 142, no. 2, pp. 399-416, 2009.

[32] J. Luedtke and S. Ahmed, "A sample approximation approach for optimization with probabilistic constraints," SIAM J. Optimiz., vol. 19, no. 2, pp. 674-699, 2008

[33] A. Ruszczyński and A. Shapiro, , A. Ruszczyński and A. Shapiro, Eds., "Stochastic programming models," in Stochastic Programming, Handbooks in Operations Research and Management Science. New York, NY, USA: Elsevier, 2003, vol. 10, pp. 1-64.

[34] V. A. Zorich, Mathematical Analysis II. New York, NY, USA: Springer, 2004.

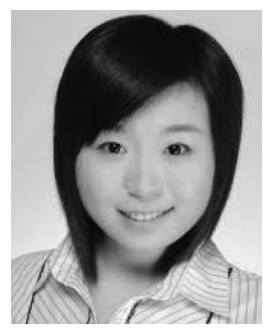

Chaoyue Zhao (S'12) received the B.S. degree in information and computing sciences from Fudan University, Shanghai, China, in 2010. She is pursuing the $\mathrm{Ph}$.D. degree in industrial and systems engineering at the University of Florida, Gainsville, FL, USA.

She is joining the Department of Industrial Engineering and Management at the Oklahoma State University, Stillwater, OK, USA, as a tenure-track assistant professor. Her research interests include stochastic optimization, robust optimization, and power grid security analysis.

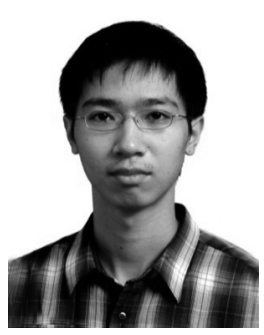

Qianfan Wang (S'10) received the B.S. degree in computer science and economics from Peking University, Beijing, China, in 2009 and the Ph.D. degree in industrial and systems engineering from the University of Florida, Gainesville, FL, USA, in 2013.

He was a visiting student in the Decision and Information Sciences Division at the Argonne National Laboratory, Argonne, IL, USA, from October 2010 to May 2011. He is currently working full-time at Alstom Grid, Redmond, WA, USA, as a power system (Market Application) engineer.

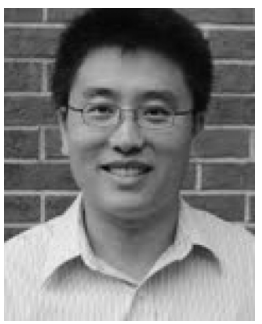

Jianhui Wang (M'07-SM'12) received the Ph.D. degree in electrical engineering from Illinois Institute of Technology, Chicago, IL, USA, in 2007.

Presently, he is a Computational Engineer with the Decision and Information Sciences Division at $\mathrm{Ar}-$ gonne National Laboratory, Argonne, IL, USA.

Dr. Wang is the chair of the IEEE Power \& Energy Society (PES) power system operation methods subcommittee and co-chair of an IEEE task force on the integration of wind and solar power into power system operations. He is an editor of the IEEE TRansactions ON POWER Systems, the IEEE TRANSACtions ON SMART GRID, an associate editor of Journal of Energy Engineering, an editor of the IEEE PES Letters, and an associated editor of Applied Energy. He is also the editor of Artech House Publishers Power Engineering Book Series and the recipient of the IEEE Chicago Section 2012 Outstanding Young Engineer Award.

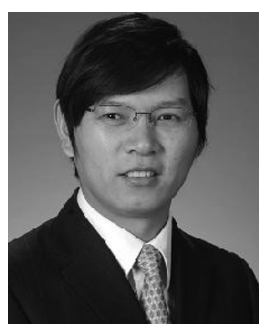

Yongpei Guan (M'10-SM'13) received the B.S. degree in mechanical engineering and the B.S. degree in economic decision science from the Shanghai Jiaotong University, Shanghai, China, in 1998, the M.S. degree in industrial engineering and engineering management from the Hong Kong University of Science and Technology, Hong Kong, China, in 2001, and the Ph.D. degree in industrial and systems engineering from the Georgia Institute of Technology, Atlanta, GA, USA, in 2005.

$\mathrm{He}$ is the Director of the Computational and Stochastic Optimization Lab at the University of Florida, Gainesville, FL, USA. His research interests include power grid security and energy policy analysis. 